\title{
WILLIAMS'S CONJECTURE IS FALSE FOR REDUCIBLE SUBSHIFTS
}

\author{
K. H. KIM AND F. W. ROUSH
}

\section{INTRODUCTION}

Subshifts of finite type (SFT's) are among the most accessible types of dynamical systems, consisting of all sequences $\left(x_{i}\right)$ from a finite alphabet that miss a finite set of forbidden words. At the same time they are very useful in the analysis of Axiom A diffeomorphisms, especially in the study of invariant measure and periodic points [Bow]. They also come up in mathematical linguistics (via regular languages) and coding theory $[\mathrm{ACH}]$.

The fundamental breakthrough in the study of SFT's was made by Williams [W]. He proved that topological conjugacy is equivalent to the matrix relation strong shift equivalence and conjectured about eighteen years ago that this was equivalent to the matrix relation shift equivalence. Shift equivalence is very tractable by matrix and algebraic methods $[\mathrm{Ba}, \mathrm{N}, \mathrm{KR}]$ and is implied by strong shift equivalence.

In this paper we show that shift equivalence does not imply strong shift equivalence for reducible SFT's. The counterexample makes essential use of an example in the preceding paper [KRW] of a mixing SFT for which the dimension representation is not surjective.

For definitions, related concepts, notation, and additional references see the preceding paper $[\mathrm{KRW}]$.

\section{CountereXample to THE Williams CONJECTURE}

The following theorem, based on a matrix argument, provides a counterexample to the Williams conjecture in the reducible case.

Theorem. Let

$$
A=\left[\begin{array}{cc}
U & 0 \\
I & U
\end{array}\right], \quad B=\left[\begin{array}{cc}
U & 0 \\
U^{20}(U-I) & U
\end{array}\right] .
$$

Then $B$ is nonnegative and $A, B$ are shift equivalent but not strong shift

Received by the editors September 18, 1990 and, in revised form, October 21, 1991.

1991 Mathematics Subject Classification. Primary 54H20.

Key words and phrases. Strong shift equivalence, shift equivalence, reducible shift, subshift of finite type.

This work was partially supported by NSF DMS-8820801 \& 9024813 . 
equivalent where

$$
U=\left[\begin{array}{llll}
0 & 0 & 1 & 1 \\
1 & 0 & 0 & 0 \\
0 & 1 & 0 & 0 \\
0 & 0 & 1 & 0
\end{array}\right], \quad I=\left[\begin{array}{llll}
1 & 0 & 0 & 0 \\
0 & 1 & 0 & 0 \\
0 & 0 & 1 & 0 \\
0 & 0 & 0 & 1
\end{array}\right]
$$

Proof. Because $U$ is primitive and $(U-I)$ multiplies the positive eigenvector of $U$ by a positive number, we know $U^{k}(U-I)$ will be positive for large $k$. A computation verifies that $k=20$ is large enough. For a particular shift equivalence of $A$ and $B$ take

$$
R=\left[\begin{array}{cc}
I & 0 \\
0 & U^{20}(U-I)
\end{array}\right], \quad S=A^{20} R^{-1}=\left[\begin{array}{cc}
U^{20} & 0 \\
20 U^{19} & U^{3}+U^{2}+U
\end{array}\right]
$$

Every strong shift equivalence gives a shift equivalence where

$$
R=\left[\begin{array}{cc}
R_{11} & 0 \\
R_{21} & R_{22}
\end{array}\right]
$$

in which the main diagonal blocks are induced by strong shift equivalences from $U$ to itself. The block $R_{12}$ must be zero for the following reasons. The matrix $U^{10}$ is positive, hence $R_{n}=B^{n} R A^{n}, S_{n}=A^{n} S B^{n}$ have all nonzero blocks positive for $n>11$. Moreover, since $\left(B^{n} R A^{n}\right)\left(A^{n} S B^{n}\right)$ is a power $B^{k}$, $k>0$, it follows that $R$ and $S$ have nonzero determinant. As above $R, S$ denote matrices in a shift equivalence from $A$ to $B$. This means that each of $R_{n}, S_{n}$ has at least three of its four blocks nonzero since $A$ divides it. From $\left(B^{n} R A^{n}\right)\left(A^{n} S B^{n}\right)=B^{k}$ we deduce that row 1 of $R$ cannot have both blocks nonzero. From $\left(A^{n} S B^{n}\right)\left(B^{n} R A^{n}\right)=A^{k}$ we deduce that column 2 of $R$ cannot have both blocks nonzero. Hence the only zero block must be in the $(1,2)$-entry.

The matrices $R_{11}$ and $R_{22}$ will represent shift equivalences from $\mathscr{S}_{U}$ to itself induced by conjugacies. In [KRW, Example 4.1] it was proved that such matrices and their product $R_{22} R_{11}^{-1}$ cannot have the form $(U-I)$.

Since products with $U^{m}$ represent compositions of an automorphism with the shift, we cannot also have $U^{j}(U-I)$ induced by an automorphism.

We may identify $\mathbf{Q}[U]$ with the algebraic number field generated by a root of the characteristic polynomial $\left(x^{4}-x-1\right)$ of $U$. We compute that $U$, $(U-I)$ are units in the algebraic number field of $U$ having inverses $\left(U^{3}-I\right)$ and $\left(U^{3}+U^{2}+U\right)$, respectively. We check that $\left(x^{4}-x-1\right)$ is irreducible by examining possible quadratic quadratic factors and rational roots.

Now we consider possible shift equivalences. Main diagonal blocks must be units. On the $(2,1)$-block we have

$$
R_{21} U+R_{22}=B_{21} R_{11}+U R_{21} .
$$

Conversely, this condition suffices for a shift equivalence over $\mathbf{Z}$ with positive main diagonal blocks since $R$ is invertible, so we can solve for $S$. We have for any $X$ in the algebraic number field (using $X U=U X$ ); 


$$
\operatorname{Tr}\left(X\left(U R_{21}-R_{21} U\right) X\right)=0 .
$$

So $\operatorname{Tr}\left(X^{2}\left(R_{22}-B_{21} R_{11}\right)\right)=0$.

Note that $B_{21} \in \mathbf{Q}(U)$. The $R_{i i}$ commute with $U$ by the equation of shift equivalence. Since $[\mathbf{Q}(U): \mathbf{Q}]=4$, it follows that $\mathbf{Q}(U)$ is its own centralizer and $R_{i i} \in \mathbf{Q}(U)$. Every element $y$ of $\mathbf{Q}(U)$ can be written as a difference of two squares

$$
X_{1}^{2}-X_{2}^{2}=\left(X_{1}-X_{2}\right)\left(X_{1}+X_{2}\right) \text {. }
$$

Hence $\operatorname{Tr}\left(y\left(R_{22}-B_{21} R_{11}\right)=0\right.$. If $R_{22}-B_{21} R_{11} \neq 0$ let $y$ be its inverse, and we have a contradiction. Thus $B_{21}$ is the ratio of the $R_{i i}$, but we have already shown this leads to a contradiction. This proves the theorem.

\title{
3. Conclusion
}

In this setting the Williams conjecture is false, but it seems that it does not fail by very much. We have shown in results to appear elsewhere that given suitable results about the irreducible case (which is separate problem) we can classify up to conjugacy reducible SFT's.

\section{ACKNOWLEDGMENT}

We would like to thank Jack Wagoner for reading the first draft and making comments and suggestions and Mike Boyle for detailed comments, and two referees for suggesting constructive changes.

\section{REFERENCES}

[ACH] R. Adler, D. Coppersmith, and M. Hassner, Algorithm for sliding block codes, IEEE Trans. Inform. Theory 29 (1983), 5-22.

[Ba] K. Baker, Strong shift equivalence of $2 \times 2$ matrices of nonnegative integers, Ergodic Theory Dynamical Systems 3 (1983), 541-558.

[Bow] R. Bowen, Markov partitions for Axiom A diffeomorphisms, Amer. J. Math. 92 (1970), $725-747$.

[KR] K. H. Kim and F. W. Roush, Some results on decidability of shift equivalence, J. Combin. Inform. System Sci. 4 (1979), 123-146.

[KRW] K. H. Kim, F. W. Roush, and J. Wagoner, Automorphisms of the dimension group and gyration numbers, J Amer. Math. Soc. 5 (1992), 191-212 (this issue).

[N] M. Nasu, Topological conjugacy for sofic systems and extensions of automorphisms of subshifts of finite type, Dynamical Systems (J. C. Alexander, ed.), Lecture Notes in Math., vol. 1342, Springer-Verlag, Heidelberg, 1988.

[W] R. F. Williams, Classification of subshifts of finite type, Ann. Math. 98 (1973), 120-153; Errata, Ann. Math. 99 (1974), 380-381.

\begin{abstract}
We show that for two subshifts of finite type having exactly two irreducible components, strong shift equivalence is not the same as shift equivalence. This refutes the Williams conjecture $[\mathrm{W}]$ in the reducible case. The irreducible case remains an open problem.
\end{abstract}

Mathematics Research Group, Alabama State University, Montgomery, Alabama 36101 